\title{
3D Breast Volume Estimation
}

\author{
Pedro F. Gouveia ${ }^{a, b}$ Hélder P. Oliveirac, d João P. Monteiro ${ }^{c}$ João F. Teixeirac

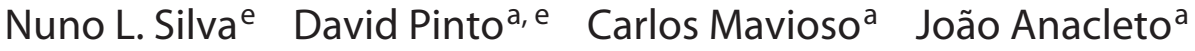 \\ Marta Martinho $^{a}$ Inês Duarte ${ }^{f}$ Jaime S. Cardoso ${ }^{c, g}$ Fatima Cardoso ${ }^{a}$ \\ Maria João Cardoso ${ }^{a, e}$ \\ ${ }^{a}$ Breast Unit, Champalimaud Clinical Center/Champalimaud Foundation, Lisbon, Portugal; ${ }^{b}$ Faculty of

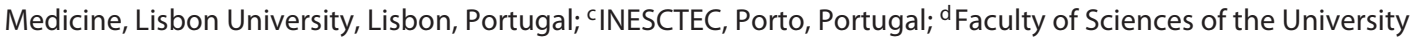

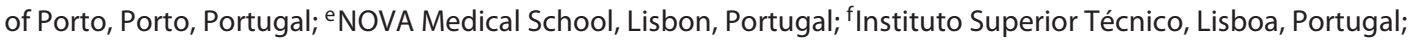 \\ ${ }^{g}$ Faculty of Engineering of the University of Porto, Porto, Portugal
}

\section{Keywords}

Breast cancer - Breast volume · 3D surface scan · Breast MRI ·

Experimental study · Surgery

\begin{abstract}
Introduction: Breast volume estimation is considered crucial for breast cancer surgery planning. A single, easy, and reproducible method to estimate breast volume is not available. This study aims to evaluate, in patients proposed for mastectomy, the accuracy of the calculation of breast volume from a low-cost 3D surface scan (Microsoft Kinect) compared to the breast MRI and water displacement technique. Material and Methods: Patients with a Tis/T1-T3 breast cancer proposed for mastectomy between July 2015 and March 2017 were assessed for inclusion in the study. Breast volume calculations were performed using a 3D surface scan and the breast MRI and water displacement technique. Agreement between volumes obtained with both methods was assessed with the Spearman and Pearson correlation coefficients. Results: Eighteen patients with invasive breast cancer were included in the study and submitted to mastectomy. The level of agreement of the 3D breast volume compared to surgical specimens and breast MRI volumes was evaluated. For mastectomy specimen volume, an aver-
\end{abstract}

(C) 2021 S. Karger AG, Basel

www.karger.com/es age (standard deviation) of $0.823(0.027)$ and $0.875(0.026)$ was obtained for the Pearson and Spearman correlations, respectively. With respect to MRI annotation, we obtained 0.828 (0.038) and 0.715 (0.018). Discussion: Although values obtained by both methodologies still differ, the strong linear correlation coefficient suggests that 3D breast volume measurement using a low-cost surface scan device is feasible and can approximate both the MRI breast volume and mastectomy specimen with sufficient accuracy. Conclusion: 3D breast volume measurement using a depth-sensor low-cost surface scan device is feasible and can parallel MRI breast and mastectomy specimen volumes with enough accuracy. Differences between methods need further development to reach clinical applicability. A possible approach could be the fusion of breast MRI and the 3D surface scan to harmonize anatomic limits and improve volume delimitation.

(C) 2021 S. Karger AG, Basel

\section{Introduction}

Breast volume estimation is considered crucial when performing breast cancer surgery planning, as the ratio between tumor volume and breast volume is the swinging pendulum between breast cancer conservative surgery

Correspondence to:

Pedro F. Gouveia, pedro.gouveia@fundacaochampalimaud.pt 


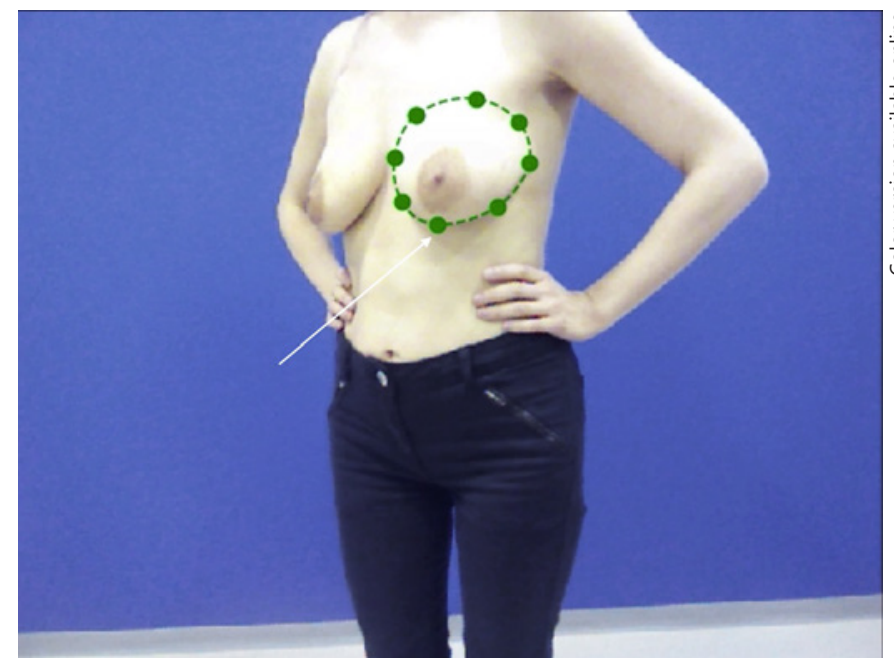

Fig. 1. 3D surface scan breast annotation with the lowest visible point (arrow).

and mastectomy $[1,2]$. In cases where mastectomy and immediate breast reconstruction are proposed, breast volume estimation could also be important to address preoperative choices in implant and autologous reconstruction.

In clinical practice, a single, easy, and reproducible method to estimate breast volume is not available. A surgeon's evaluation is made by subjective observation of the patient's anatomy. Several methods have been described to calculate breast volume; the anthropomorphic method $[3,4]$ uses standardized measurements of the thoracic region, while the Archimedean method [5] is based on water displacement techniques. The Grossman-Roudner measurement device is an alternative, with an adjustable geometrical conical disk into which the breast is placed [6]. Mammograms are a readily available option and have been used with multiple formulas to estimate breast volume [7-10], including measuring and recording height, diameter, and compression thickness. Computed tomography $(\mathrm{CT})[11,12]$ and magnetic resonance imaging (MRI) $[8,13,14]$ are also possible, but have the disadvantage of not being routinely performed for breast cancer diagnosis, thus adding extra cost. 3D photography has been used for volume and projection estimation, mainly for esthetic surgery [15]. The 3D surface scan is a more recent viable option $[13,16-18]$, but commercially proposed options are still very expensive and its clinical applicability has not been properly tested. The purpose of this work was to evaluate the accuracy of breast volume calculation, in patients proposed for mastectomy, with a

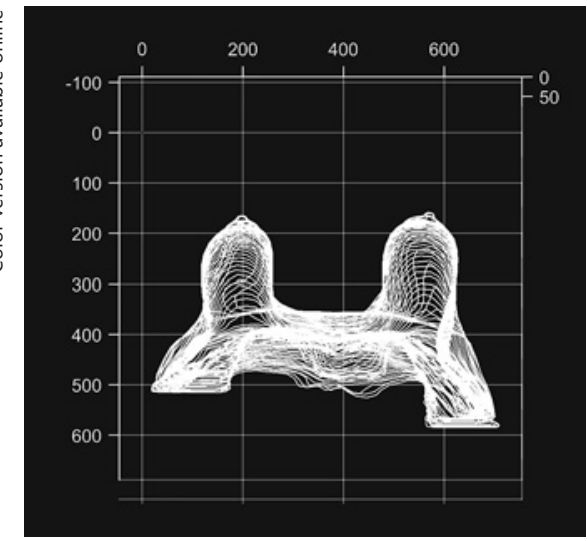

Fig. 2. 3D breast information after segmenting all MRI slices.

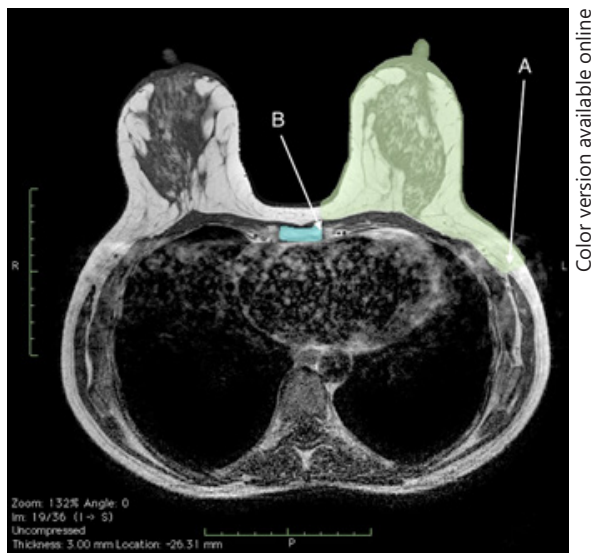

Fig. 3. Breast MRI annotation with external (anterior border latissimus dorsi muscle, A) and internal (sternum external border, B) limits.

low-cost 3D surface scan (Microsoft Kinect) compared to the breast MRI and water displacement technique (the Archimedean method).

\section{Material and Methods}

Patients with Tis/T1-T3 breast cancer proposed for mastectomy treatment at the Champalimaud Clinical Center, Portugal, between July 2015 and March 2017, were assessed for inclusion in the study (according to the mastectomy specimen volume calculation at baseline). Contraindications included T4 cancer, inflammatory carcinoma, and breast recurrence post-breast conservative treatment. All patients were proposed for image acquisition with breast MRI and 3D surface scanning.

The $3 \mathrm{D}$ surface scan was conducted with the patient in the standing position, with hands on hips, capturing the patient's torso by means of the Microsoft Kinect Sensor ${ }^{\circledR}$ v1.0. Scan acquisition was performed with the sensor in a fixed position with the patient rotating through the sagittal axis plane. 
Table 1. Volumetric estimation using MRIs, 3D surface scans, and mastectomy surgical specimens

\begin{tabular}{|c|c|c|c|c|c|c|}
\hline \multirow[t]{2}{*}{ ID } & \multirow{2}{*}{$\begin{array}{l}\text { Type of } \\
\text { mastectomy }\end{array}$} & \multirow{2}{*}{$\begin{array}{l}\text { Mastectomy } \\
\text { specimen volume, } \\
\mathrm{mL}\end{array}$} & \multirow{2}{*}{$\begin{array}{l}\text { MRI breast } \\
\text { volume, } \mathrm{mL}\end{array}$} & \multicolumn{3}{|c|}{$3 \mathrm{D}$ volume calculation } \\
\hline & & & & rater $\mathrm{A}$ & rater $\mathrm{B}$ & rater $\mathrm{C}$ \\
\hline 1 & NSSM & 160 & 437.08 & 301.90 & 249.47 & 250.72 \\
\hline 2 & WM & 600 & $1,773.52$ & 962.75 & 984.07 & 970.34 \\
\hline 3 & $\mathrm{M}$ & 350 & 930.08 & 491.75 & 431.83 & 611.39 \\
\hline 4 & $\mathrm{M}$ & 210 & 831.13 & 376.78 & 397.19 & 432.52 \\
\hline 5 & NSSM & 290 & 773.78 & 578.72 & 568.75 & 573.56 \\
\hline 6 & M & 400 & 911.41 & 594.21 & 542.00 & 507.60 \\
\hline 7 & WM & 230 & $1,663.55$ & 418.94 & 450.57 & 472.08 \\
\hline 8 & WM & 300 & 607.51 & 509.19 & 499.31 & 481.03 \\
\hline 9 & WM & 475 & $1,037.82$ & 804.26 & 666.93 & 726.77 \\
\hline 10 & NSSM & 95 & 178.27 & 121.11 & 100.26 & 92.07 \\
\hline 11 & $\mathrm{M}$ & 600 & 936.35 & 685.29 & 632.22 & 757.76 \\
\hline 12 & M & 120 & 295.04 & 279.70 & 255.94 & 258.92 \\
\hline 13 & $\mathrm{M}$ & 250 & $1,110.42$ & 530.61 & 494.34 & 382.55 \\
\hline 14 & M & 850 & $1,208.54$ & 675.35 & 607.09 & 550.99 \\
\hline 15 & M & 225 & 643.61 & 497.31 & 497.31 & 515.50 \\
\hline 16 & NSSM & 230 & 808.63 & 956.37 & 502.51 & 501.66 \\
\hline 17 & WM & 470 & $1,090.94$ & $1,045.89$ & 845.54 & 975.75 \\
\hline 18 & WM & 625 & $1,681.71$ & $1,268.97$ & $1,193.97$ & $1,191.53$ \\
\hline 19 & WM & 1,000 & $2,233.42$ & $1,587.35$ & $1,585.27$ & $1,625.01$ \\
\hline
\end{tabular}

M, modified radical mastectomy; WM, wise pattern mastectomy; NSSM, nipple skin-sparing mastectomy.

Annotation of the 3D surface scan data was performed by 3 independent raters (A-C), i.e., breast surgeons (Fig. 1). The annotators performed this task with no knowledge of the other measures (i.e., specimen and breast MRI volumes).

Volume calculations were performed considering correspondences between positions in color (RGB) - depth camera space and world space, paired with a convex hull approach to estimate posterior wall of the $3 \mathrm{D}$ breast surface scan.

MRI with gadolinium contrast was performed according to institutional protocol, with the patient in the prone position with arms up; annotation, segmentation, and volume computation of the MRI tissue portions with T1 image sequences (Fig. 2) were performed and validated by 2 radiologists using Horos $R$ software v2.4.0 (breast contour, breast tissue, pectoralis major muscle, anterior border of the latissimus dorsi muscle as the breast external limit, sternum lateral border as the breast internal limit, and clavicle) (Fig. 3).

Surgical specimens were measured, orientated, and weighted in the operating room. Intraoperative volume measurement was performed using the water displacement technique (Archimedean method).

\section{Statistical Analysis}

Data are presented as mean and standard deviation (SD) or relative and absolute frequencies. The agreement between the volumes obtained with both methods was assessed with the Pearson and Spearman correlation coefficients. The coefficient of reliability for each rater (intrarater reliability) was calculated. All statistical analyses were calculated with SPSS software v20.0.

3D Breast Volume

\section{Results}

\section{Patients}

Eighteen patients with invasive breast cancer were included in the study and submitted to mastectomy (bilateral in 1 case), either nipple-sparing (3 patients and 4 specimens), Wise pattern (7 patients), or modified radical mastectomy (8 patients). Their mean age was 57.4 (range 30-86) years.

\section{Volumetric Analysis}

The average volume of the 19 mastectomy specimens by water displacement was $393.7 \mathrm{~mL}$ (range 95-1,000 $\mathrm{mL}$ ) (Table 1). Average calculated volumes for breast MRI annotation were 1,008.04 mL (range 178.27-2,233.42 $\mathrm{mL}$ ). The average breast volume of the $3 \mathrm{D}$ reconstructed model was 667.71 (range 121.11-1,587.50), 605.50 (range 100.27-1,585.27), and 625.15 (range 92.07-1,625.01) mL for rater $\mathrm{A}, \mathrm{B}$, and $\mathrm{C}$, respectively.

To validate the methodology and the coherence between each rater for the $3 \mathrm{D}$ breast surface scan annotation, the level of agreement between them was evaluated by means of the Pearson and Spearman correlation coefficients; an average (SD) of 0.960 (0.020) and 0.914 
Table 2. Level agreement between all methods used for breast volume calculation (surface scan with each rater vs. mastectomy specimen and breast MRI)

\begin{tabular}{lll}
\hline & Pearson & Spearman \\
\hline Rater A vs. mastectomy specimen & 0.79 & 0.85 \\
Rater B vs. mastectomy specimen & 0.84 & 0.90 \\
Rater C vs. mastectomy specimen & 0.83 & 0.87 \\
Rater A vs. breast MRI & 0.79 & 0.72 \\
Rater B vs. breast MRI & 0.86 & 0.73 \\
Rater C vs. breast MRI & 0.83 & 0.69 \\
\hline
\end{tabular}

(0.046), respectively, was obtained, proving that each rater annotated coherently.

The level of agreement of the raters' calculation of values from the surface scan, surgical specimen, and MRI annotation was also evaluated (Table 2). For mastectomy specimen volume, an average (SD) of $0.823(0.027)$ and 0.875 (0.026) was obtained for the Pearson and Spearman correlations, respectively. Concerning MRI annotation, we obtained $0.828(0.038)$ and $0.715(0.018)$.

The metrics used, as well as the average and SD values obtained, demonstrate a very high consistency by the 3 raters in the baseline volume annotation and when this was compared with the volumes obtained from mastectomy specimens and the MRI data (Table 2). This high level of agreement provided us with great confidence in the designed methodology.

\section{Discussion}

Estimating breast volume with different modalities has long been a research focus for improved breast cancer surgery planning, with breast MRI having been considered the gold standard [13]. However, since 3D surface scanning has become available, it is reported to be a valid method for estimating breast volume.

Breast MRI volume calculation is based on breast contour segmentation in each of the MRI slices, and it relies on easily recognizable anatomical structures as reference points. In our series of patients, deploying all 3 methods, the average volume calculated from breast MRI was higher $(1,008.04 \mathrm{~mL})$ than that calculated from the 3D surface scan $(667.71 \mathrm{~mL})$ and the Archimedean $(393.7 \mathrm{~mL})$ method. These results are in line with previous reports, but the mean deviation is higher in our series $[10,13,19]$. The discrepancy in this overestimation is easily explained by annotation constraints of breast MRI external and inter- nal limits. The anterior border of the latissimus dorsi muscle used as an anatomical reference to mark the external limits of the breast does not always correlate with the visible external contouring using the $3 \mathrm{D}$ surface scan (Fig. 3). This means that a middle point between the anterior border of the latissimus dorsi muscle and the true external breast contour is very hard to determine, and is thus not a reproducible reference. Previous authors did not describe their methodology or differ on how to define breast boundaries applied to MRI segmentation [10, 13, $19,20]$. The same applies to the inner breast limit and the superior pole limit. This is an important limitation to the automation of breast MRI volume estimation.

Breast volume estimation with the $3 \mathrm{D}$ surface scan method between the 3 raters had a highly significant interrater reliability coefficient (Pearson $=0.960$; Spearmann $=0.875)$, confirming previous reports $[17,18]$. The average volume calculation from the different raters varied between a maximum of $667.71 \mathrm{~mL}$ (rater A) and a minimum of $605.50 \mathrm{~mL}$ (rater B). Chest wall estimation is the main constrain when using this method to calculate breast volume since the convex hull approach may not always accurately detect the anterior chest wall curvature, and it cannot simulate the major and minor pectoralis muscles. As the inframammary fold is not always visible, adopting the lowest visible point in the breast is a viable option (Fig. 1). The upper pole limit can also be difficult to define with a $3 \mathrm{D}$ surface scan reconstruction, despite a good agreement between different raters being achieved.

The average volume calculation of the mastectomy surgical specimens attained the lowest estimation of all the methods, i.e., $393.7 \mathrm{~mL}$. Residual breast tissue after mastectomy may explain these differences, along with different mastectomy techniques that will resect different quantities of skin.

Although values obtained by both methodologies still differ, the strong linear correlation coefficient suggests that $3 \mathrm{D}$ breast volume measurement using a low-cost surface scanning device, the Microsoft Kinect 1, is feasible and can approximate both the MRI breast volume and mastectomy specimen with sufficient accuracy. These results are aligned with previous reports, and the need for further developments is also conclusion reached by all authors [13, 18, 20-22]. It is well accepted that the influence of the patient's position on volume calculation is an important limitation when correlating different volume calculations with different imaging modalities. This means that shape analysis needs to be added in the assessment of morphological results to address proper surgical planning. A possible approach could be the fusion of a breast 
MRI to a 3D surface scan of the patient's torso, to achieve proper synchronization and a volumetric alignment of the 2 methods [23]. A breast MRI pose-related transformation from the prone to the standing position for acquisition needs to be performed to match the 3D scan shape [23]. This development could pave the way for new clinical applications like breast cancer surgery with augmented reality technology [24].

\section{Conclusion}

$3 \mathrm{D}$ breast volume measurement using a depth sensor low-cost surface scan device like Microsoft Kinect is feasible, and it paralleled MRI breast and mastectomy specimen volumes by obtaining an average Pearson coefficient $>0.8$ in both cases. Differences between methods need further developments to reach clinical applicability in breast cancer surgery with improved planning. A possible approach could be the fusion of a breast MRI to a $3 \mathrm{D}$ surface scan to harmonize anatomic limits and improve volume delimitation.

\section{Statement of Ethics}

This study (reference: Breast 3D) was approved by the Champalimaud Foundation Ethics Committee. Participants' written informed consent in compliance with the Helsinki Declaration was received. Participants' written informed consent to publish photos and radiologic images relating to their person was obtained.

\section{Conflict of Interest Statement}

Fátima Cardoso reports personal financial interest in form of consultancy role for: Amgen, Astellas/Medivation, AstraZeneca, Celgene, Daiichi-Sankyo, Eisai, GE Oncology, Genentech, GlaxoSmithKline, Macrogenics, Medscape, Merck-Sharp, Merus BV, Mylan, Mundipharma, Novartis, Pfizer, Pierre-Fabre, prIME Oncology, Roche, Samsung Bioepis, Sanofi, Seagen, Teva.

All authors declare that they have no known competing financial interests or personal relationships that could have appeared to influence the work reported in this paper.

\section{Funding Sources}

This research did not receive any specific grant from funding agencies in the public, commercial, or not-for-profit sectors.

\section{Author Contributions}

Study concept: P.F.P.G. Study design: P.F.P.G. and H.P.O. Data acquisition: P.F.P.G., N.L.S., D.P., C.M., J.A., and M.M. Quality control of data and algorithms: P.F.P.G., H.P.O., J.P.M., J.F.T., and I.D. Data analysis and interpretation: P.F.P.G., H.P.O., J.P.M., J.F.T., and I.D. Statistical analysis: P.F.P.G., H.P.O., and I.D. Manuscript preparation and editing: P.F.P.G. and H.P.O. Manuscript review: J.S.C., F.C., and M.J.C.

\section{References}

1 Clough KB, Kaufman GJ, Nos C, Buccimazza I, Sarfati IM. Improving breast cancer surgery: a classification and quadrant per quadrant atlas for oncoplastic surgery. Ann Surg Oncol. 2010 May;17(5):1375-91.

2 Vos EL, et al. Preoperative prediction of cosmetic results in breast conserving surgery. J Surg Oncol. 2015;111(2):178-84.

3 Westreich M. Anthropomorphic breast measurement: protocol and results in 50 women with aesthetically perfect breasts and clinical application. Plast Reconstr Surg. 1997 Aug;100(2):468-79.

4 Mahmoud EO, Hosnia AM, Bothaina A, Mahmoud ES. Assessment of the breast volume by a new simple formula. Indian J Plast Surg. 2006;39(01):13-6.

5 Bouman FG. Volumetric measurement of the human breast and breast tissue before and during mammaplasty. Br J Plast Surg. 1970 Jul;23(3):263-4.
6 Grossman AJ, Roudner LA. A simple means for accurate breast volume determination. Plast Reconstr Surg. 1980 Dec;66(6):851-2.

7 Katariya RN, Forrest AP, Gravelle IH. Breast volumes in cancer of the breast. Br J Cancer. 1974 Mar;29(3):270-3.

8 Bulstrode N, Bellamy E, Shrotria S. Breast volume assessment: comparing five different techniques. Breast. 2001 Apr;10(2):117-23.

9 Fung JT, Chan SW, Chiu AN, Cheung PS, Lam SH. Mammographic determination of breast volume by elliptical cone estimation. World J Surg. 2010 Jul;34(7):1442-5.

10 Kayar R, Civelek S, Cobanoglu M, Gungor O, Catal H, Emiroglu M. Five methods of breast volume measurement: a comparative study of measurements of specimen volume in 30 mastectomy cases. Breast Cancer (Auckl). 2011 Mar;5:43-52.

11 Nelson TR, Cerviño LI, Boone JM, Lindfors KK. Classification of breast computed tomography data. Med Phys. 2008 Mar;35(3):107886.
12 Erić M, Anderla A, Stefanović D, Drapšin M. Breast volume estimation from systematic series of CT scans using the Cavalieri principle and $3 \mathrm{D}$ reconstruction. Int $\mathrm{J}$ Surg. 2014;12(9):912-7.

13 Kovacs L, Eder M, Hollweck R, Zimmermann A, Settles M, Schneider A, et al. Comparison between breast volume measurement using $3 \mathrm{D}$ surface imaging and classical techniques. Breast. 2007 Apr;16(2):137-45.

14 Yoo A, Minn KW, Jin US. Magnetic resonance imaging-based volumetric analysis and its relationship to actual breast weight. Arch Plast Surg. 2013 May;40(3):203-8.

15 Galdino GM, Nahabedian M, Chiaramonte M, Geng JZ, Klatsky S, Manson P. Clinical applications of three-dimensional photography in breast surgery. Plast Reconstr Surg. 2002 Jul;110(1):58-70. 
16 Passalis G, et al. Noninvasive automatic breast volume estimation for post-mastectomy breast reconstructive surgery. Proceedings of the 25th Annual International Conference of the IEEE Engineering in Medicine and Biology Society (IEEE Cat. No.03CH37439). 2003.

17 Losken A, Seify H, Denson DD, Paredes AA Jr, Carlson GW. Validating three-dimensional imaging of the breast. Ann Plast Surg. 2005 May;54(5):471-6.

18 Kovacs L, Eder M, Hollweck R, Zimmermann A, Settles M, Schneider A, et al. New aspects of breast volume measurement using 3-dimensional surface imaging. Ann Plast Surg. 2006 Dec;57(6):602-10.
19 Wang C, Luan J. Magnetic Resonance Imaging Versus 3-Dimensional Laser Scanning for Breast Volume Assessment After Breast Reconstruction. Ann Plast Surg. 2018 May;80(5): 592.

20 Göpper MW, Neubauer J, Kalash Z, Stark GB, Simunovic F. Improved accuracy of breast volume calculation from 3D surface imaging data using statistical shape models. PLoS One. 2020 Nov;15(11):e0233586.

21 Losken A, Fishman I, Denson DD, Moyer HR, Carlson GW. An objective evaluation of breast symmetry and shape differences using 3-dimensional images. Ann Plast Surg. 2005 Dec;55(6):571-5.
22 Koch MC, Adamietz B, Jud SM, Fasching PA, Haeberle L, Karbacher S, et al. Breast volumetry using a three-dimensional surface assessment technique. Aesthetic Plast Surg. 2011 Oct;35(5):847-55.

23 Bessa S, Gouveia PF, Carvalho PH, Rodrigues C, Silva NL, Cardoso F, et al. 3D digital breast cancer models with multimodal fusion algorithms. Breast. 2020 Feb;49:281-90.

24 Gouveia PF, Costa J, Morgado P, Kates R, Pinto $\mathrm{D}$, Mavioso $\mathrm{C}$, et al. Breast cancer surgery with augmented reality. Breast. 2021 Apr;56:14-7. 